\title{
Neutrophil extracellular traps promote macrophage pyroptosis in sepsis
}

Linsong Chen ${ }^{1,2}$, Yanfeng Zhao ${ }^{1}$, Dengming Lai ${ }^{3}$, Peng Zhang ${ }^{1}$, Yang Yang ${ }^{1}$, Yuehua $\mathrm{Li}^{2,4}$, Ke Fei $^{1}$, Gening Jiang ${ }^{1}$ and Jie Fan ${ }^{1,2,4,5}$

\begin{abstract}
In response to infection, polymorphonuclear neutrophils (PMN) are recruited in the infectious sites, and employ three major strategies to fight against the microbes including phagocytosis, degranulation, and neutrophil extracellular traps (NETs). NETs are a meshwork of chromatin fibers mixed with granule-derived antimicrobial peptides and enzymes, which trap and kill the bacteria extracellularly. In this study, by using a mouse sepsis model, we identified a novel mechanism by which NETs induce macrophage $(M \phi)$ pyroptosis, a caspase-1-dependent regulated cell death. We show that NET-derived HMGB1, acting through RAGE and dynamin-dependent signaling, triggers an intra-M $\phi$ cascade of molecular events including cathepsin B (CatB) release from the ruptured lysosomes, followed by pyroptosome formation and caspase-1 activation, and subsequent $M \phi$ pyroptosis. The study further demonstrates that $M \phi$ pyroptosis augments inflammatory responses following sepsis. These findings shed light on the proinflammatory role of NETs in mediating PMN-M interaction, which therefore influences the progress of inflammation following infection.
\end{abstract}

\section{Introduction}

Sepsis, a leading cause of human death worldwide, is characterized by excessive inflammation in response to infection ${ }^{1}$. During septic peritonitis, polymorphonuclear neutrophils (PMN) and monocytes are recruited in the peritoneal cavity, and the latter differentiate into inflammatory macrophages $(\mathrm{M} \phi)^{2}$. The interaction between $\mathrm{PMN}$ and $\mathrm{M} \phi$ has been suggested as an important factor that regulates inflammation following trauma, hemorrhagic shock, and endotoxemia, as well as other pathological conditions ${ }^{3-7}$. For instance, we have reported that exosomes released from the hemorrhagic shock-activated alveolar M $\phi$ promote PMN necroptosis in the lungs ${ }^{5}$. Others have demonstrated that neutrophil extracellular

\footnotetext{
Correspondence: Ke Fei (ffeike@126.com) or Gening Jiang (jgnwp@aliyun.com) or Jie Fan (jif7@pitt.edu)

${ }^{1}$ Department of Thoracic Surgery, Shanghai Pulmonary Hospital, Tongji

University School of Medicine, Shanghai 200433, China

${ }^{2}$ Department of Surgery, University of Pittsburgh School of Medicine,

Pittsburgh, PA 15213, USA

Full list of author information is available at the end of the article

Edited by H.-U. Simon.
}

traps (NETs) acting through inducing cytokine production from $M \phi$ promote inflammation and the development of atherosclerosis ${ }^{7}$. However, in sepsis, the role of the interaction between PMN and M $\mathrm{M}$ in the development of inflammation and the underlying mechanism remains unclear.

In response to microbial infection, one of the defensing mechanisms of the host is to release nuclear contents of the PMN into the extracellular space to trap and kill the microbes, known as NETs. Since first discovered in $2004^{8}$, NETs have been recognized as an important strategy of the host immune system to respond against infections ${ }^{9}$. NETs are composed of decondensed chromatin decorated with granular and cytoplasmic proteins, which may serve as danger-associated molecular patterns (DAMPs) playing critical role in the progression of host inflammation ${ }^{10,11}$.

High-mobility group box 1 (HMGB1), a highly conserved nuclear protein widely present in the nucleus and cytoplasm of nearly all cell types, is the prototypic DAMP molecule when released into the extracellular space ${ }^{12}$. Our previous studies reported that HMGB1 plays a

\section{(c) The Author(s) 2018}

(c) (i) Open Access This article is licensed under a Creative Commons Attribution 4.0 International License, which permits use, sharing, adaptation, distribution and reproduction cc) in any medium or format, as long as you give appropriate credit to the original author(s) and the source, provide a link to the Creative Commons license, and indicate if changes were made. The images or other third party material in this article are included in the article's Creative Commons license, unless indicated otherwise in a credit line to the material. If material is not included in the article's Creative Commons license and your intended use is not permitted by statutory regulation or exceeds the permitted use, you will need to obtain permission directly from the copyright holder. To view a copy of this license, visit http://creativecommons.org/licenses/by/4.0/. 
critical role in inducing pyroptosis of $\mathrm{M} \phi$ and vascular endothelial cell $(\mathrm{EC})^{13,14}$. Pyroptosis is a caspase-1dependent form of regulated cell death, which is usually triggered by various pathological stimuli, i.e., intracellular pathogens and extracytoplasmic stimuli ${ }^{15}$. The characteristics of pyroptosis include cell swelling, rapid plasma membrane rupture, and release of proinflammatory contents $^{16}$. Pyroptotic cell-released danger signals or DAMP molecules enhance inflammatory responses ${ }^{17}$.

In this study, we identified a novel mechanism of PMN-M $\phi$ interaction in sepsis. We demonstrate in the mouse cecal ligation and puncture (CLP) sepsis model that PMN through ejecting NETs induce peritoneal M $\phi$ pyroptosis. We further confirm that HMGB1 released from NETs acting through the receptor for advanced glycation end products (RAGE) initiates dynamindependent signaling pathway that includes cathepsin $B$ (CatB) activation, pyroptosome formation, and caspase-1 activation, which in turn leads to $\mathrm{M} \phi$ pyroptosis. These findings suggest a previously unidentified pathway of $\mathrm{PMN}-\mathrm{M} \phi$ cross-talk, which causes enhanced $\mathrm{M} \phi$ death and subsequent exaggerated post-sepsis inflammation.

\section{Results}

\section{Sepsis induces peritoneal $\mathbf{M} \phi$ pyroptosis}

In CLP-induced sepsis, we found that a considerable part of the peritoneal $\mathrm{M} \phi(\mathrm{PM} \phi)$ underwent cell death. As shown in Fig. 1, mice subjected to CLP exhibited a gradual increase in $\mathrm{PM} \phi$ death. The PM $\phi$ death, as defined as double staining of Annexing $\mathrm{V}$ and 7-AAD, reached a peak $(\sim 36 \%)$ at $18 \mathrm{~h}$ after CLP. In order to determine the type of $\mathrm{PM} \phi$ death, $\mathrm{PM} \phi$ collected from the peritoneal lavage fluid (PLF) were further detected for nuclear fragmentation, caspase- 1 activation, and the characteristics of pyroptosis, by staining the cells with TMR-Cell Death Reagent and Alexa Flour 488-labeled caspase-1 FLICA, and measured by flow cytometry. We observed $\sim 14 \%$ pyroptotic PM $\phi$ at $18 \mathrm{~h}$ after CLP (Fig. 1b). These results suggest that pyroptosis is responsible for $\sim 39 \%$ of the PM $\phi$ death following sepsis.

Interestingly, we further observed NET formation in PLF, associated with the exhibition of PM $\phi$ pyroptosis following CLP. PLF samples were collected from mice that were subjected to either CLP or sham operation for 0 to $24 \mathrm{~h}$, and each of the PLF samples was divided into two aliquots for florescence microscopy of NETs and PM $\phi$ pyroptosis, respectively. Using SYTOX green staining of DNA and florescence microscopy, we detected in PLF a progressive increase in NET formation, which started at as early as $3 \mathrm{~h}$ and reached a peak at $6 \mathrm{~h}$ after CLP, and remained at a high level for at least $24 \mathrm{~h}$ (Fig. 1c, e). Associated with the formation of NETs, PM $\phi$ pyroptosis occurrence starts at $12 \mathrm{~h}$ after CLP (Fig. 1d). Intraperitoneal injection (i.p.) of Cl-Amidine (Cl-Ad, $50 \mathrm{mg} / \mathrm{kg} \mathrm{B}$.
W.), an inhibitor of NET formation by suppressing peptidylarginine deiminase $4(\mathrm{PAD} 4)^{18,19}$, at $30 \mathrm{~min}$ prior to CLP prevented NET formation following sepsis (Fig. 1e). Notably, the suppression of NET formation was associated with the decrease in $\mathrm{PM} \phi$ pyroptosis at $18 \mathrm{~h}$ after CLP (Fig. 1f). These results led us to determine the causal role of NETs in PM $\phi$ pyroptosis.

\section{NETs induce PM $\phi$ pyroptosis}

To investigate a direct role of NETs in regulating $\mathrm{M} \phi$ death, we collected peritoneal PMN, which were recruited into the peritoneal cavity in response to i.p. injection of 9\% casein, and stimulated the PMN in vitro with PMA $(50 \mathrm{nM})$ for $2 \mathrm{~h}$ to induce NETs ${ }^{20}$. The NET formation was confirmed by SYTOX green and histone double staining, and florescence microscopy, as shown in Fig. 2a. The induced NETs in the culture medium supernatant were then co-cultured with normal bone marrow-derived macrophages (BMDM) for up to $24 \mathrm{~h}$, followed by the detection of BMDM pyroptosis. As shown in Fig. 2b, NETs induced BMDM pyroptosis, which reached a peak at $12 \mathrm{~h}$ after the co-culture; however, the medium supernatant from unstimulated PMN did not cause BMDM pyroptosis.

These results suggest that NET formation in the peritoneal cavity following sepsis is responsible for $\mathrm{PM} \phi$ pyroptosis.

\section{NET-derived HMGB1 induces PM $\phi$ pyroptosis}

Our previous studies showed that HMGB1 can induce cell pyroptosis $^{13,14}$, which led us to hypothesize that HMGB1 released from the NETs might serve as a critical mediator to induce $\mathrm{PM} \phi$ pyroptosis, as considering that NETs consist of decondensed chromatin, and HMGB1 is one of the chromatin proteins. We first determined whether the NET component includes HMGB1. Peritoneal PMN were isolated and stimulated with PMA $(50 \mathrm{nM})$ for up to $4 \mathrm{~h}$ to induce NET formation in the presence and absence of NET inhibitor Cl-Ad $(200 \mu \mathrm{M})^{21}$, and then HMGB1 in the PMN medium supernatant was measured by western blotting. As shown in Fig. 3a, b, at $2 \mathrm{~h}$ after PMA stimulation, with NET formation, HMGB1 started to increase in the supernatant; however, the NET inhibitor Cl-Ad prevented HMGB1 increase in the supernatant. These in vitro observations were recapitulated in vivo. HMGB1 level in PLF was significantly elevated at $6 \mathrm{~h}$ after CLP, and maintained at a high level for at least 24h (Fig. 3c). This elevation in the HMGB1 level was suppressed by a pre-treatment with i.p. injection of NET inhibitor Cl-Ad (Fig. 3d). These results suggest that NET formation associates with HMGB1 release.

To determine whether the NET-derived HMGB1 induces PM $\phi$ pyroptosis, we subjected wild-type (WT) and $\mathrm{HMGB1}^{-I-}$ mice to CLP for $18 \mathrm{~h}$, and then measured 


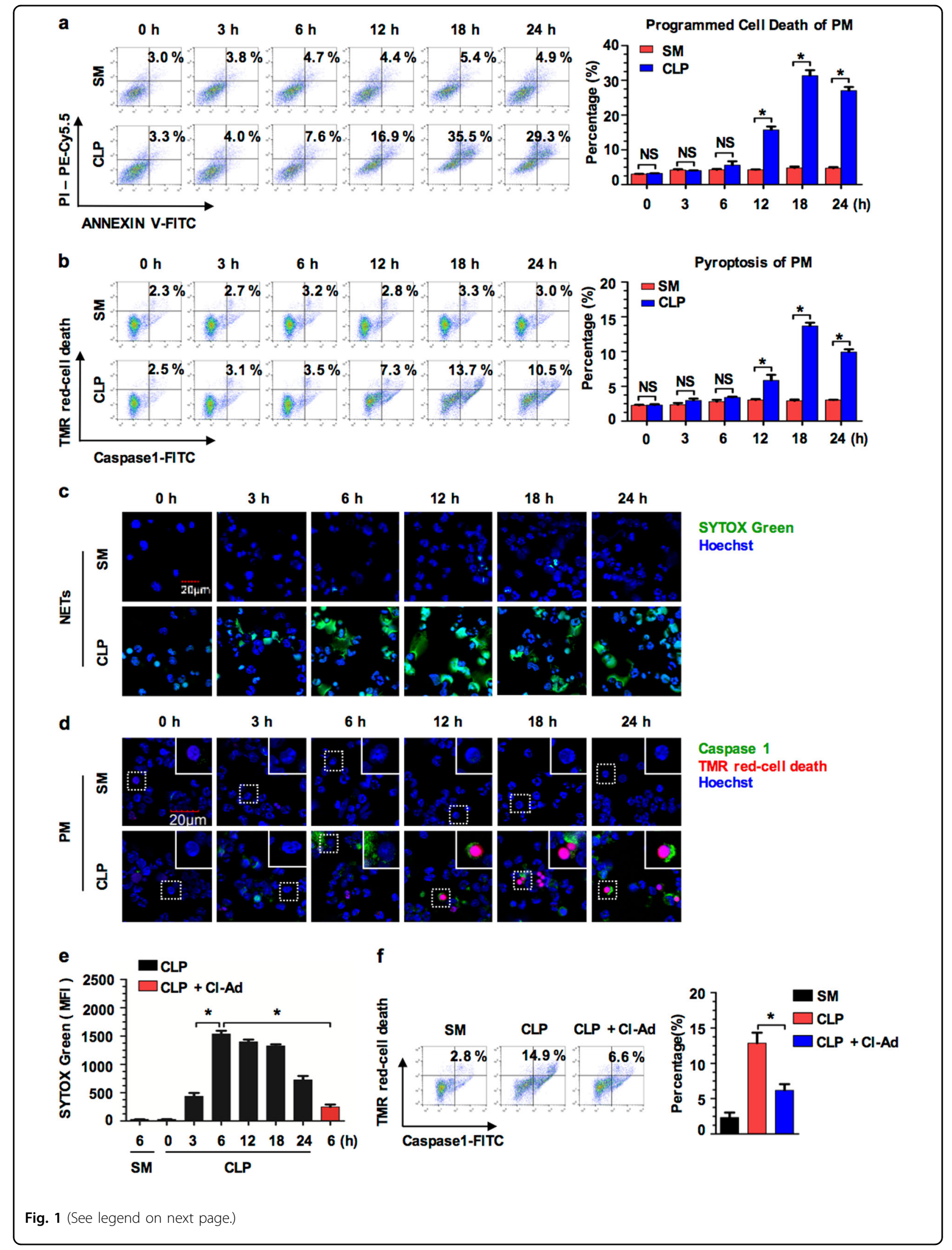


PM $\phi$ pyroptosis. HMGB1 deficiency significantly decreased the PM $\phi$ pyroptosis, as compared to that in the WT mice (Fig. 3e), although NET formation in the CLP groups was not affected by HMGB1 deficiency (Fig. 3f). To specifically define the role of the NET-derived HMGB1 in inducing $M \phi$ pyroptosis, we induced NETs in WT and HMGB1 $^{-1-}$ PMN in vitro and co-cultured the NETs with WT normal BMDM for $12 \mathrm{~h}$. As shown in Fig. 3g, HMGB1 deficiency significantly attenuates the NET-induced BMDM pyroptosis, as compared to the group treated with WT NETs. These results demonstrate an important role of NET-released HMGB1 in inducing M $\phi$ pyroptosis.

\section{RAGE-dynamin signaling mediates NET-induced PM $\phi$ pyroptosis}

To determine whether NET-induced M $\phi$ pyroptosis following sepsis is a specific receptor-dependent event, we isolated BMDM from WT, $\mathrm{RAGE}^{-1-}, \mathrm{TLR}^{-1-}$, and TLR9 ${ }^{-1-}$ mice, and then treated the cells with NETs (or the medium supernatant of the unstimulated PMN as a control) for $12 \mathrm{~h}$. The results showed that RAGE deficiency effectively prevented $M \phi$ pyroptosis induced by NETs, whereas the genetic deletion of TLR4 or TLR9 failed to block $M \phi$ pyroptosis (Fig. 4a). We further subjected $\mathrm{RAGE}^{-/-}$mice to CLP and observed that RAGE deficiency significantly prevented PM $\phi$ pyroptosis at $18 \mathrm{~h}$ after CLP, as compared to that in the WT mice (Fig. 4b), although there was no significant change in NET formation between these two groups (Fig. 4c).

Our previous in vitro study has shown that RAGE-dynamin signaling mediates HMGB1-induced M $\phi$ pyroptosis $^{13}$. However, this mechanism has yet to be determined in an in vivo sepsis model. To elucidate the role of RAGE-dynamin signaling in NET-induced PM $\phi$ pyroptosis, we applied dynamin inhibitor dynasore $(30 \mu \mathrm{g} /$ $\mathrm{ml}$ ) in the NET-BMDM co-culture system, and found that dynasore exhibited a suppressive effect on the NETinduced BMDM pyroptosis (Fig. 4d). Collectively, these results show that NET-derived HMGB1 mediates $M \phi$ pyroptosis via a RAGE-dynamin pathway.
Lysosome destabilization and cathepsin B activation are
required for pyroptosome formation and PM $\phi$ pyroptosis It has been reported that pyroptosome, a complex including adaptor protein apoptosis-associated specklike protein containing a CARD (ASC), also known as ASC focus, recruits pro-caspase-1, resulting in its activation and proteolysis of the mature form via neighboring activated caspase- 1 proteins ${ }^{22}$. Our previous study showed that pyroptosome, rather than inflammasome, mediates HMGB1-induced M $\phi$ pyroptosis via lysosome enzyme cathepsin B (CatB)-dependent pathway $^{13}$. To determine whether CatB pathway contributes to NET-induced M $\phi$ pyroptosis, we treated WT BMDM with NETs that were induced from WT or $\mathrm{HMGB}^{-/-}$ PMN in the presence or absence of dynasore $(30 \mu \mathrm{g} / \mathrm{ml})$ for $9 \mathrm{~h}$, followed by measuring the lysosome integrity and CatB activation in the BMDM using fluorescence-tagged DQ ovalbumin and Magic Red CatB detection reagent, respectively. The WT NETs induced lysosome rupture and CatB activation in WT M $\phi$, while dynasore effectively blocked these effects (Fig. 5a, b). HMGB1 ${ }^{-/-}$NETs did not induce the lysosome destabilization, and CatB activation, as shown in Fig. 5a, b. In addition, WT NETs failed to induce lysosome rupture and $\mathrm{CatB}$ activation in RAGE deficiency Mф (Fig. 5a, b). Furthermore, we subjected the WT, HMGB1 ${ }^{-1-}$, and $\mathrm{RAGE}^{-/-}$mice to CLP for $12 \mathrm{~h}$, and detected lysosome destabilization and CatB activation in PMф. As shown in Fig. 5c, d, CLP caused the lysosome rupture and $\mathrm{CatB}$ activation in the $\mathrm{PM} \phi$ from WT mice, but not in the PM $\phi$ from the $\mathrm{HMGB1}^{-1-}$ and $\mathrm{RAGE}^{-1-}$ mice.

To determine whether pyroptosome formation is implicated in the $\mathrm{M} \phi$ pyroptosis induced by NET-derived HMGB1, we stimulated WT BMDM with WT NETs or $\mathrm{HMGB1}^{-1-}$ NETs in the presence or absence of CatB inhibitor CA-074-me $(10 \mu \mathrm{M})^{13}$, followed by visualizing the ASC focus using florescence-tagged ASC antibody and confocal microscopy, and detecting caspase- 1 activation by western blotting. Figure. 5e, f shows that WT NETs induced ASC foci formation and caspase- 1 activation, whereas CatB

(see figure on previous page)

Fig. 1 Sepsis induces peritoneal $\mathbf{M} \boldsymbol{\phi}$ (PM $\boldsymbol{\phi}$ ) pyroptosis. a-c Mice were subjected to CLP for up to $24 \mathrm{~h}$. a Regulated PM $\phi$ death was assessed by PE-Annexin-V and 7-AAD double-staining using flow cytometry. $\mathbf{b}$ The pyroptosis of PM $\phi$ was assessed by Cell Death Reagent-TMR and Alexa Fluor 488-labeled caspase-1 FLICA double-staining by flow cytometry. $\mathbf{c}$, $\mathbf{d}$ Peritoneal lavage fluid (PLF) was collected from mice that were subjected to either CLP or sham for $0,3,6,12,18$, and $24 \mathrm{~h}$, and then each of the PLF samples was divided into two aliquots for florescence microscopy to determine NETs (c) and macrophage pyroptosis (d), respectively. NET formation in the supernatant of PLF was assessed by staining with DNA-binding dye, SYTOX Green. PM $\phi$ were stained with Cell Death Reagent-TMR (red) and Alexa Fluor 488-labeled caspase-1 FLICA (green); the double-stained pyroptotic cells were detected by confocal microscopy. e Mice were subjected to CLP for up to $24 \mathrm{~h}$, and Cl-Ad (50 mg/kg B.W.) in some experiments was injected into the peritoneal cavity at 30 min prior to CLP. Quantification of NET formation in the supernatant of PLF was assessed by staining with DNA-binding dye, SYTOX Green. $\mathbf{f}$ Mice were subjected to CLP for $18 \mathrm{~h}$ with or without Cl-Ad pre-treatment. The pyroptosis of PM $\phi$ was assessed by Cell Death Reagent-TMR and Alexa Fluor 488-labeled caspase-1 FLICA double-staining by flow cytometry. All results are representative of the five independent experiments, and graphs depict the values of the mean and the S.E.M. ${ }^{*} P<0.05$, as compared between two groups. NS, no significant difference. Higher magnification images for the selected area are shown in the boxed insets (original magnification $\times 600$ ) 

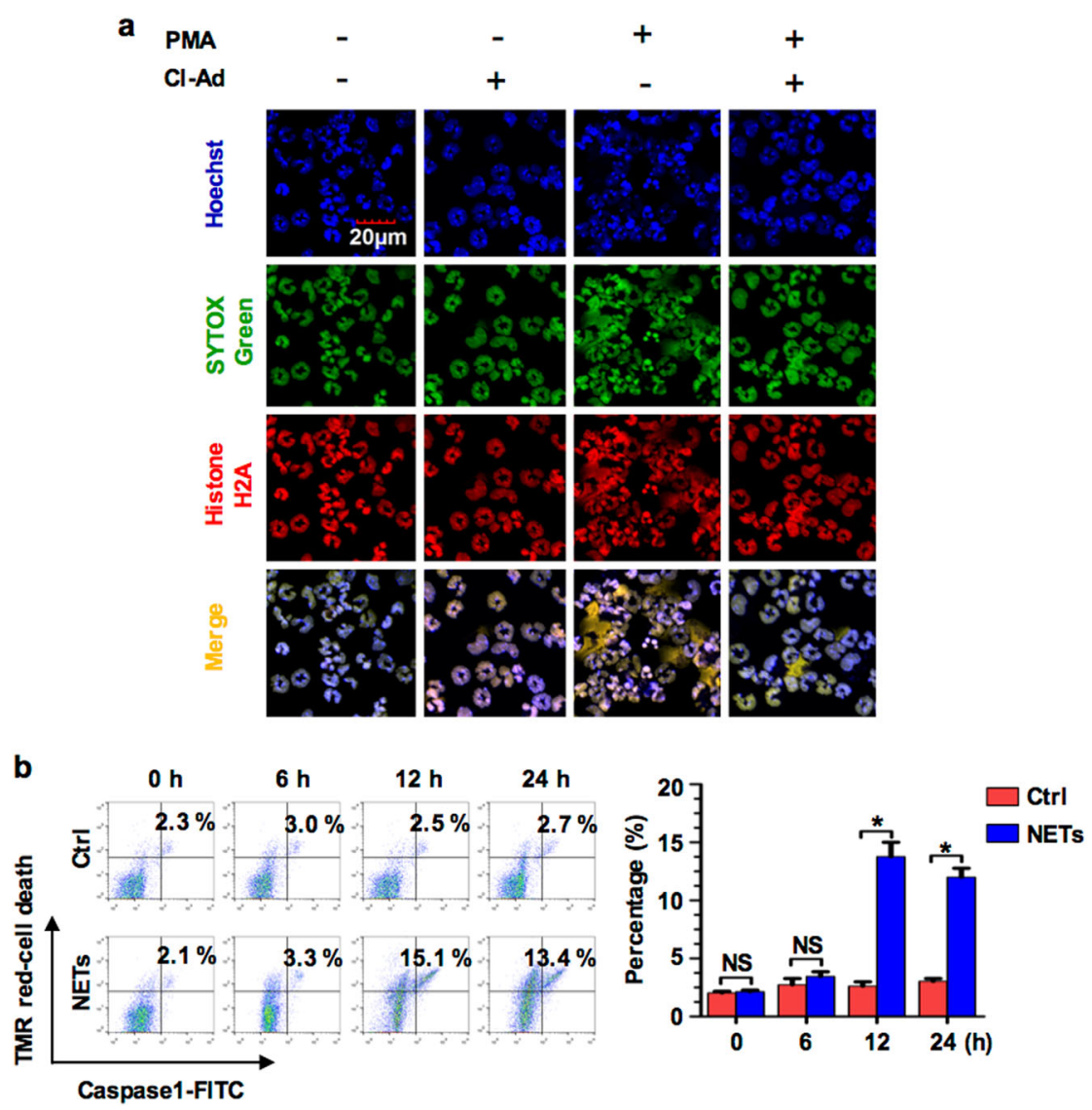

Fig. 2 NETs induce PM $\boldsymbol{\phi}$ pyroptosis . a Peritoneal PMN were pretreated with or without $\mathrm{Cl}-\mathrm{Ad}(200 \mu \mathrm{M})$ for 30 min, and then were stimulated by PMA $(50 \mathrm{nM})$ for $2 \mathrm{~h}$ to induce NET formation that was confirmed by SYTOX green and histone 2A double staining, and florescence microscopy. Fluorescent images were obtained by confocal microscopy (original magnification $\times 600$ ). b BMDM were incubated with NETs for up to $24 \mathrm{~h}$. BMDM pyroptosis was assessed by Cell Death Reagent-TMR and Alexa Fluor 488-labeled caspase-1 FLICA by flow cytometry. All the results are representative of the five independent experiments, and graphs depict the values of the mean and the S.E.M. ${ }^{*} P<0.05$, compared between two groups. NS, no significant difference

inhibitor prevented these effects of WT NETs; HMGB1 ${ }^{-/-}$ NETs failed to induce ASC foci formation and caspase-1 activation. Taken together, these results suggest that NETderived HMGB1 mediates M $\phi$ pyroptosis through RAGE-CatB-ASC-Caspase1 signaling pathway.

\section{NET-induced $M \phi$ pyroptosis enhances inflammatory responses}

We have previously reported that pyroptotic EC increased the inflammatory cytokine expression in nonpyroptotic cells, suggesting a proinflammatory effect of the pyroptotic cells ${ }^{14}$. To determine the role of M $\phi$ pyroptosis in acute inflammatory response, WT, RAGE ${ }^{-1-}$, and caspase $1^{-/-}$mice were subjected to CLP for $18 \mathrm{~h}$ with or without i.p of NET inhibitor Cl-Ad (50 mg/kg B.W.), and then tumor necrosis factor- $\alpha$ (TNF- $\alpha$ ) and the interleukin-1 $\beta$ (IL-1 $\beta$ ) concentrations in PLF were measured by enzyme-linked immunosorbent assay (ELISA).
As shown in Fig. 6a, b, TNF- $\alpha$ and IL-1 $\beta$ levels significantly increased in the PLF collected from WT mice following CLP, whereas the genetic deletion of either RAGE or caspase-1 markedly decreased the cytokines release in response to CLP. Furthermore, NET inhibitor $\mathrm{Cl}$-Ad suppressed the release of TNF- $\alpha$ and IL-1 $\beta$ in PLF after sepsis. In order to determine that the observed influences are specifically derived from $\mathrm{M} \phi$ pyroptosis, we first treated WT BMDM and $\mathrm{RAGE}^{-1-}$ BMDM (as a negative control) with NETs for $12 \mathrm{~h}$ to induce pyroptosis, and then co-cultured the pyroptotic BMDM with normal WT BMDM in the Transwell for $6 \mathrm{~h}$ to assess the effect of pyroptotic $\mathrm{M \phi}$ on normal neighboring $\mathrm{M \phi}$. As shown in Fig. 6c, d, WT normal M $\phi$ co-cultured with pyroptotic BMDM (NET-treated WT BMDM) exhibited a significant increase in TNF- $\alpha$ and IL- $1 \beta$ mRNA expression; however, WT normal M $\phi$ co-cultured with NET-treated RAGE ${ }^{-/-}$ BMDM, which were not induced to pyroptosis, presented 

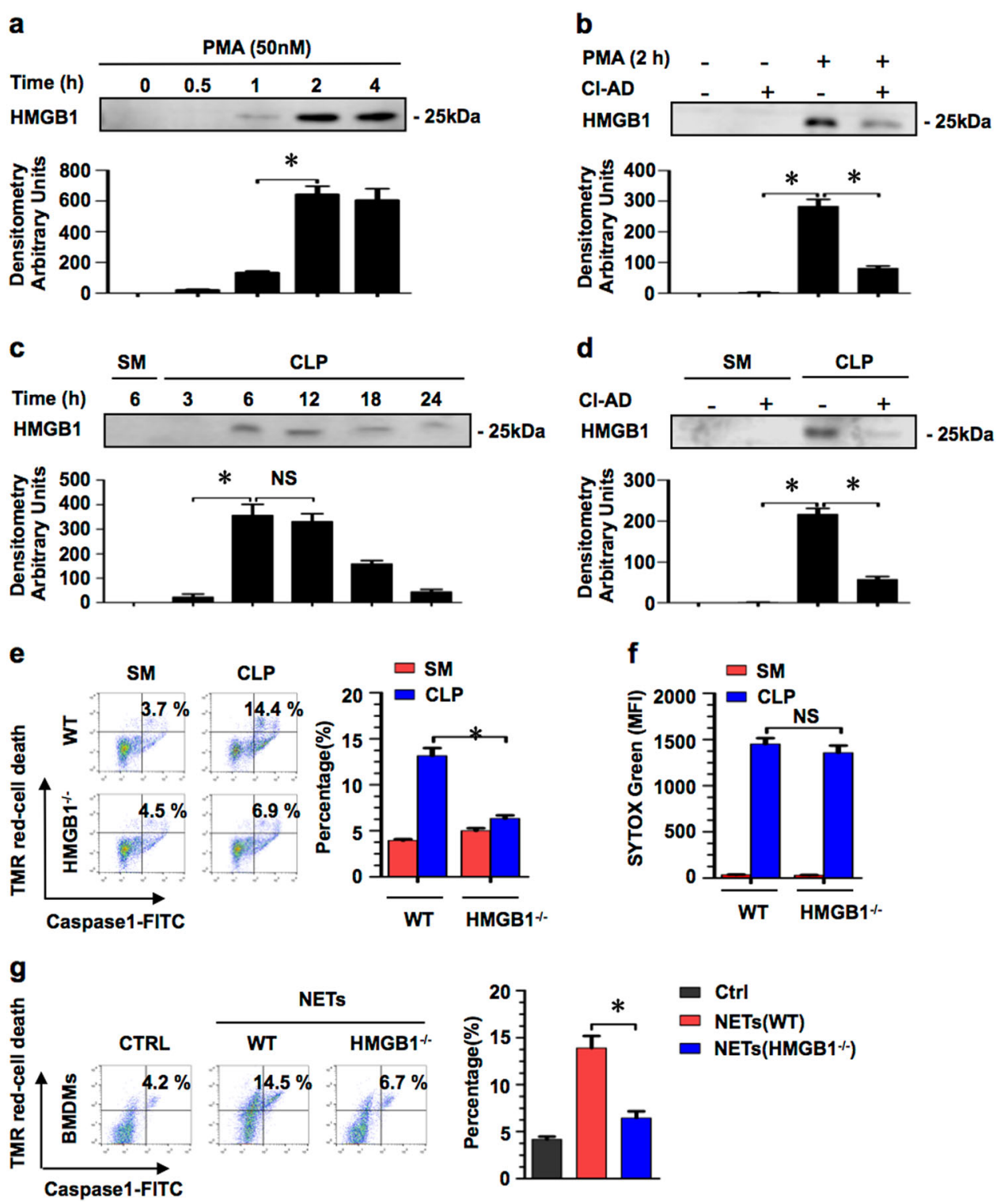

Fig. 3 NET-derived HMGB1 induces PM $\boldsymbol{\phi}$ pyroptosis. a Peritoneal PMN were stimulated with PMA (50 nM) for up to $4 \mathrm{~h}$ to induce NETs, and HMGB1 in the medium supernatant was measured by western blotting. b Peritoneal PMNs stimulated with PMA (50 nM) for $2 \mathrm{~h}$ with or without Cl-Ad $(200 \mu M)$. HMGB1 was measured in the medium supernatant by western blotting. c Mice were subjected to CLP for up to $24 \mathrm{~h}$, and HMGB1 was measured in the PLF supernatant by western blotting. $\mathbf{d}$ Mice were subjected to CLP for $6 \mathrm{~h}$ with or without i.p. injection of $\mathrm{Cl}-\mathrm{Ad}(50 \mathrm{mg} / \mathrm{kg}$ B.W.), and HMGB1 was measured in the PLF supernatant by western blotting. e WT and HMGB1 ${ }^{-1}$ mice were subjected to CLP for $18 \mathrm{~h}$, and then PM $\phi$ pyroptosis was assessed by Cell Death Reagent-TMR and Alexa Fluor 488-labeled caspase-1 FLICA double-staining by flow cytometry. $\mathbf{f}$ WT and $\mathrm{HMGB1}^{-\prime-}$ mice were subjected to CLP for $6 \mathrm{~h}$. Quantification of NET formation in the supernatant of PLF was assessed by SYTOX Green. $\mathbf{g}$ NETs induced from WT and HMGB1 ${ }^{-1}$ PMN were co-cultured with WT normal BMDM for $12 \mathrm{~h}$. BMDM pyroptosis was assessed by Cell Death Reagent-TMR and Alexa Fluor 488-labeled caspase-1 FLICA double-staining by flow cytometry. All the results are representative of the five independent experiments, and graphs depict the values of the mean and the S.E.M. ${ }^{*} P<0.05$, compared between the two groups. NS, no significant difference

a much lower level of TNF- $\alpha$ and IL-1 $\beta$ mRNA expression.

\section{Discussion}

Ungoverned inflammation is responsible for high morbidity and mortality during sepsis ${ }^{23}$. The interaction between innate immune cell populations, especially the interaction between PMN and M $\phi$, plays a critical role in the development of inflammation ${ }^{24}$. In this study, we demonstrate a previously unidentified role for PMN-M $\phi$ interaction in promoting inflammation in sepsis. We showed that while NETs function as an anti-bacteria 

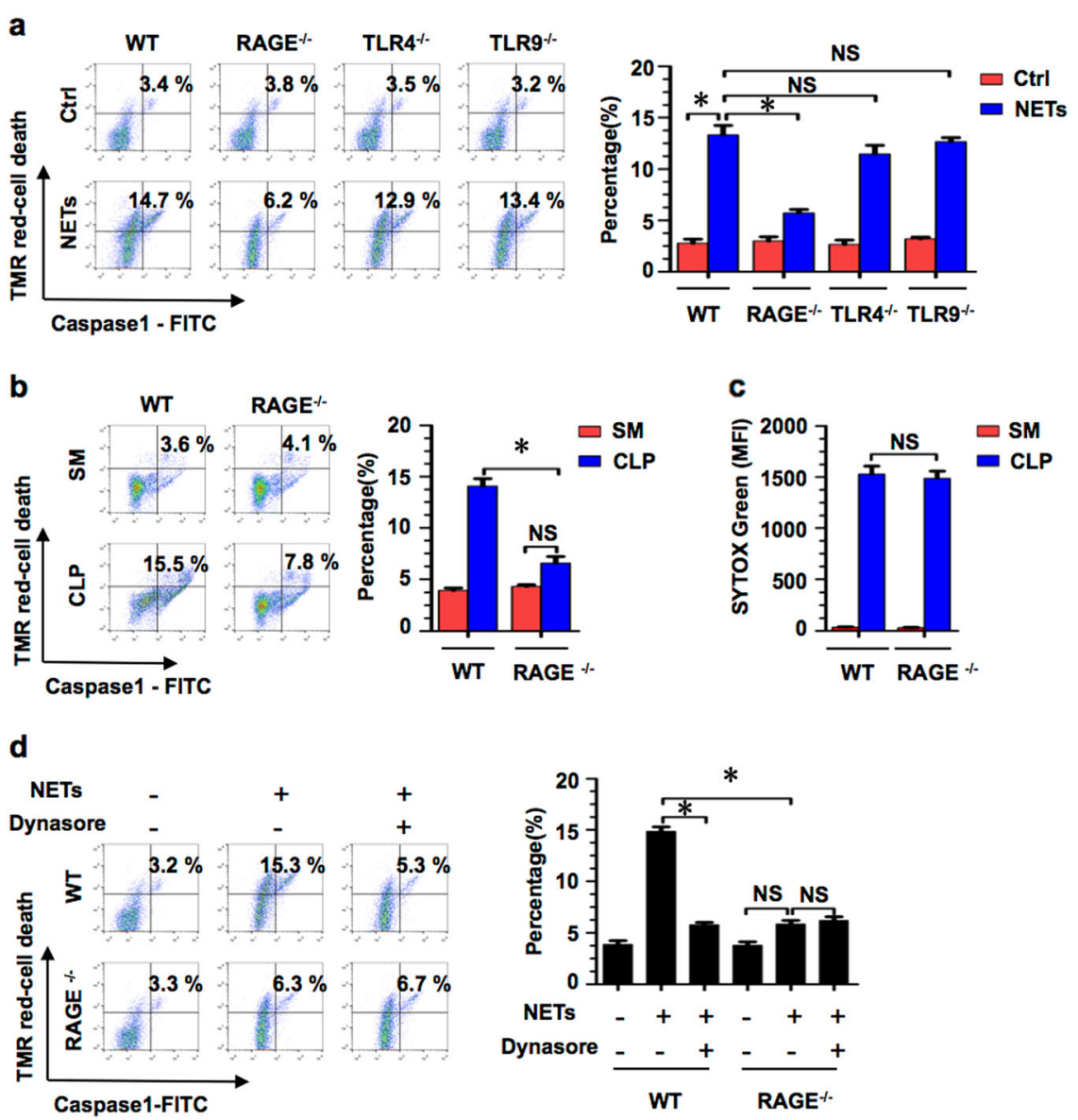

Fig. 4 RAGE-dynamin signaling mediates NET-induced PM $\boldsymbol{\phi}$ pyroptosis. a BMDM derived from WT, RAGE ${ }^{-1-}, \mathrm{TLR}^{-1-}$, and $\mathrm{TLR}^{-/-}$mice were stimulated by NETs for $12 \mathrm{~h}$. BMDM pyroptosis was assessed by Cell Death Reagent-TMR and Alexa Fluor 488-labeled caspase-1 FLICA double-staining by flow cytometry. $\mathbf{b}$ WT and RAGE ${ }^{-1-}$ mice were subjected to CLP for $18 \mathrm{~h}$ and the PM $\phi$ pyroptosis was assessed by Cell Death Reagent-TMR and Alexa Fluor 488-labeled caspase-1 FLICA double-staining by flow cytometry. c WT and RAGE ${ }^{-1}$ mice were subjected to CLP for $6 \mathrm{~h}$, and then the quantification of NETs in the PLF supernatant was measured by the mean fluorescence intensity (MFI) of SYTOX green. $\mathbf{d}$ BMDM derived from WT and RAGE $^{-1-}$ mice were stimulated by NETs with or without dynasore $(30 \mu \mathrm{g} / \mathrm{ml})$, and BMDM pyroptosis was assessed by Cell Death Reagent-TMR and Alexa Fluor 488-labeled caspase-1 FLICA double-staining by flow cytometry. All the results are representative of the five independent experiments, and graphs depict the values of the mean and the S.E.M. ${ }^{*} P<0.05$, compared between the two groups. NS, no significant difference

mechanism, they can also induce $M \phi$ death, particularly pyroptosis, which in turn enhances inflammation.

In response to infection, PMN are recruited into the infectious sites and employ three major strategies to fight against the microbes including phagocytosis, degranulation, and NETs. NETs are a meshwork of chromatin fibers mixed with granule-derived antimicrobial peptides and enzymes, such as elastase, cathepsin G, and myeloperoxidase $^{8}$. Accompanied with NET ejection, a range of molecules are released into the extracellular space. Some endogenous molecules serve as DAMPs and exert the role of proinflammatory cytokine in the development of inflammatory diseases including acute organ injury ${ }^{7,25-28}$. However, the mechanism by which NET-derived DAMPs promote inflammation, remains poorly addressed. In this study, we revealed that HMGB1 released with NETs mediates $M \phi$ death, particularly pyroptosis, thereby amplifying the inflammation. We demonstrate that NET formation was associated with increased extracellular level of HMGB1 and inhibition of NET formation by $\mathrm{Cl}$ Ad decreased the extracellular HMGB1 in vivo and in vitro. Although an elevated basal level of HMGB1 in PLF was detected after CLP, NET-caused further increase in the HMGB1 level in PLF seems required for PM $\phi$ pyroptosis, since inhibition of NET formation significantly decreased M $\phi$ pyroptosis. The role of NET-released HMGB1 in inducing $M \phi$ pyroptosis was further evidenced by the observation that NETs formed from $\mathrm{HMGB1}^{-1-}$ PMN failed to induce M $\phi$ pyroptosis.

As a DAMP molecule, HMGB1 implicates as an endogenous proinflammatory mediator to induce nuclear factor- $\mathrm{kB}$ activation and augments the secretion of TNF- 


\section{a}
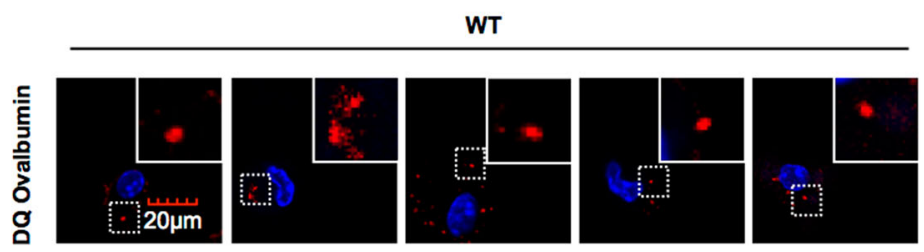

RAGE- -

NETs (WT)

NETs (HMGB1-l) -

Dynasore

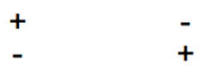

b

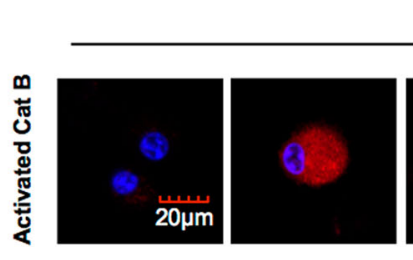

WT
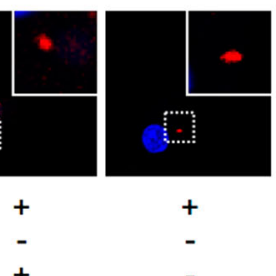

$+$

-
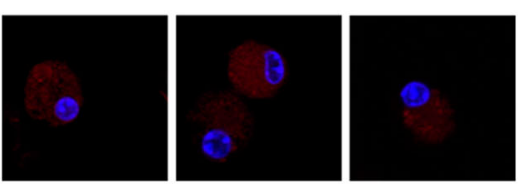

RAGE--

NETs (WT)

NETS (HMGB1 ${ }^{-1}$ ) -

Dynasore

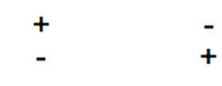

C
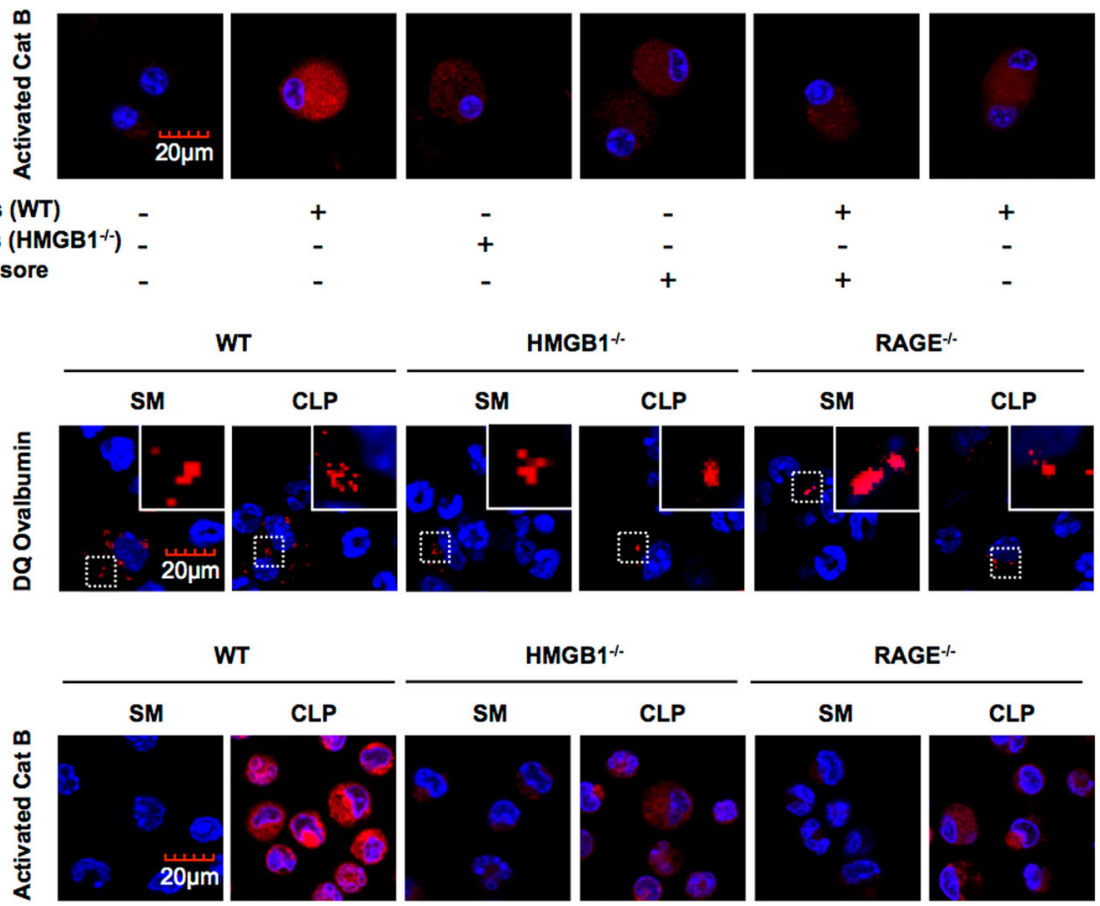

HMGB1-

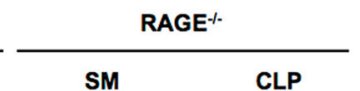

CLP

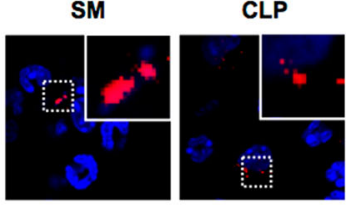

d

CLP

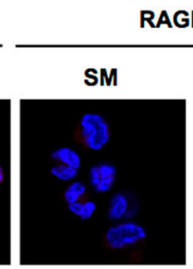

RAGE- ${ }^{-1-}$
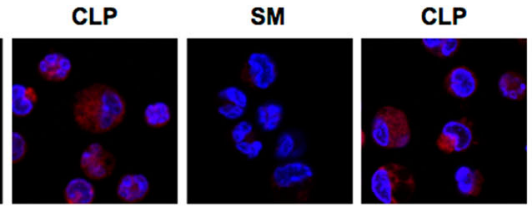

e

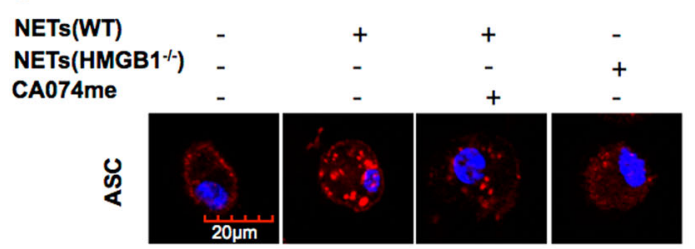

f

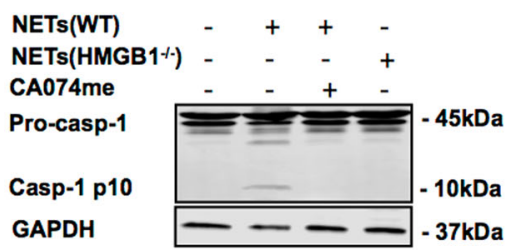

Fig. 5 Lysosome destabilization and Cathepsin B activation are required for pyroptosome formation and PM $\phi$ pyroptosis. a, b BMDM derived from the WT and the RAGE ${ }^{-1-}$ mice were stimulated by NETs derived from WT and HMGB1 ${ }^{-1-}$ mice with or without dynasore ( $\left.30 \mu \mathrm{g} / \mathrm{ml}\right)$ for $9 \mathrm{~h}$. a The cells were incubated with DQ ovalbumin (red) for $1 \mathrm{~h}$ to visualize lysosome integrity by confocal microscopy. $\mathbf{b}$ The cells were stained with Magic Red CatB detection reagent (red) to visualize the activated CatB by confocal microscopy. $\mathbf{c}, \mathbf{d}$ WT, HMGB1 ${ }^{-/-}$, and RAGE ${ }^{-/-}$mice were subjected to CLP for 12 h. c The PM $\phi$ were incubated with DQ ovalbumin (red) for $1 \mathrm{~h}$ to visualize lysosome integrity. $\mathbf{d}$ PM $\phi$ were stained with Magic Red CatB detection reagent (red) to visualize the activated CatB by confocal microscopy. e, f BMDM derived from WT mice were stimulated by NETs derived from WT and $\mathrm{HMGB}_{1}{ }^{-1-}$ mice for $9 \mathrm{~h}$ with or without CA-074-me $(10 \mu \mathrm{M})$. e ASC foci were stained to visualize pyroptosome formation; $\mathbf{f}$ Caspase-1 cleavage $(10 \mathrm{kDa})$ in the cell lysates was detected by western blotting. All the results are representative of the five independent experiments. Higher magnification images for the selected area are shown in the boxed insets (original magnification $\times 600$ )

$\alpha$, IL-1 $\beta$, IL-6, and IL-12 $29-31$. Our previous studies demonstrated a novel function of HMGB1 in inducing M $\phi$ and vascular EC pyroptosis in non-infectious settings ${ }^{13,14}$. We demonstrated that HMGB1 acting through RAGE and dynamin-dependent signaling initiated HMGB1 endocytosis, which in turn induced cell 

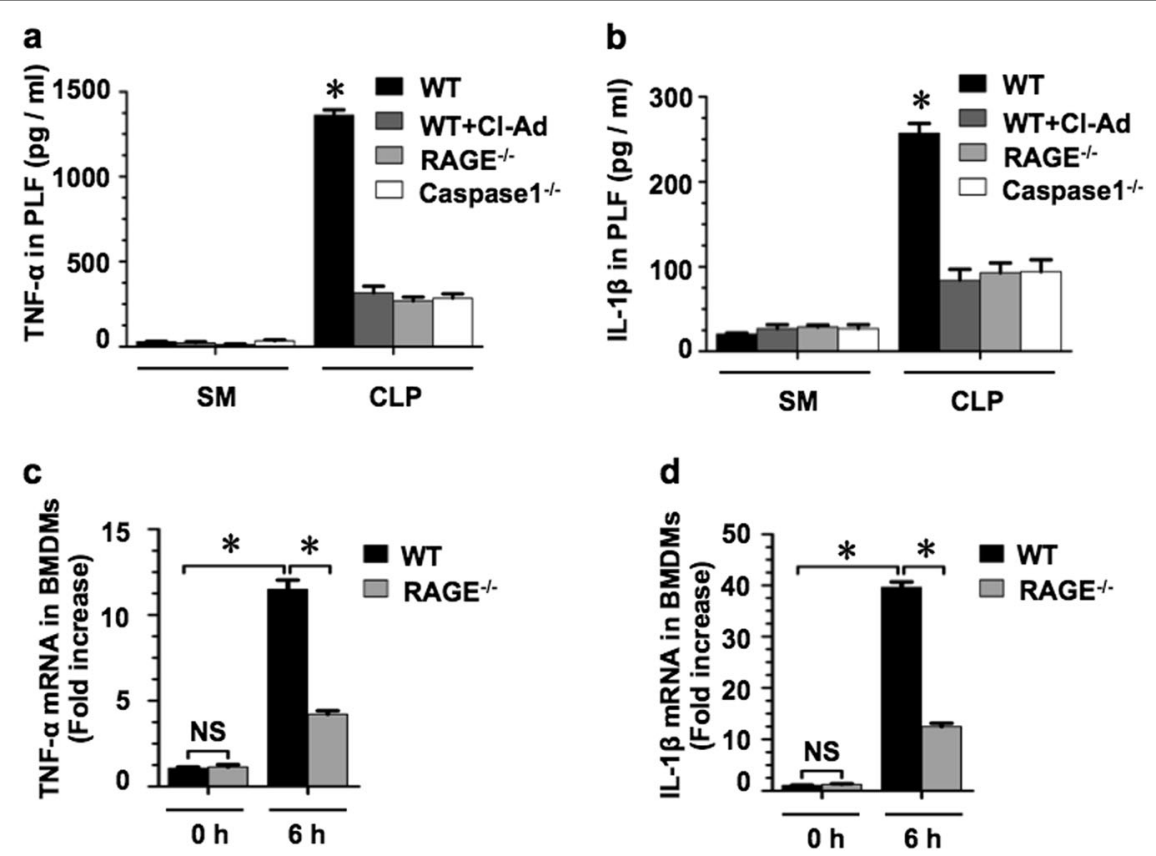

Fig. 6 NET-induced $\mathbf{M} \boldsymbol{\phi}$ pyroptosis enhances inflammatory responses. $\mathbf{a}, \mathbf{b}$ WT and RAGE ${ }^{-1-}$ mice were subjected to CLP for $18 \mathrm{~h}$; the TNF- $a$ and IL-1 $\beta$ levels in the PLF were measured by ELISA. c, d BMDM derived from WT and RAGE ${ }^{-1-}$ mice were treated with NETs for $12 \mathrm{~h}$ to induce pyroptosis in the upper well of the Transwell, followed by co-incubating with untreated WT BMDM, which were in the bottom well of the Transwell, for additional $6 \mathrm{~h}$. TNF- $a$ and IL-1 $\beta$ mRNA levels in the WT BMDM in the bottom well were then measured by qRT-PCR. The graphs depict the values of the mean and the S.E.M. $n=5,{ }^{*} P<0.05$, compared between two groups. NS, no significant difference

pyroptosis. The endocytosis of HMGB1 triggered a cascade of molecular events including CatB release from the ruptured lysosomes, followed by pyroptosome formation and caspase- 1 activation. However, it was not clear whether this pathway was also valid in sepsis. The current study not only explored the source of HMGB1 that induces $M \phi$ pyroptosis, but also validated the pathway in sepsis model. We demonstrate in this study that suppression of HMGB1-RAGE binding or its downstream events including RAGE deletion, inhibition of dynamin, CatB, and caspase- 1 activation prevent $M \phi$ pyroptosis. These findings support a causal role for HMGB1-RAGE-dynamin signaling in the induction of cell pyroptosis, which is valid in both infectious and noninfectious conditions.

Pyroptosis was primarily defined as caspase-1dependent death form ${ }^{32}$. Activated caspase-1 cleaves Gasdermin-D (GSDMD) to generate a mature GSDMD that induces pore formation on the membrane, resulting in cell swelling, plasma membrane rupture, and release of proinflammatory intracellular contents ${ }^{33}$. Release of intracellular molecules from the pyroptotic cells into the extracellular space is thought to be a proinflammatory event, which would result in exaggerated inflammation. In the current study, we determined the influence of $M \phi$ pyroptosis in the host inflammatory response. We found that the levels of TNF- $\alpha$ and IL- $1 \beta$ in PLF are significantly elevated in response to CLP; however, suppressing $M \phi$ pyroptosis by inhibiting NET formation or deletion of RAGE or caspase-1 markedly decreased the levels of TNF$\alpha$ and IL-1 $\beta$ in PLF following CLP. In vitro study further showed that pyroptotic M $\phi$ was able to induce TNF- $\alpha$ and IL- $1 \beta$ expression in normal $M \phi$, and the induction is mediated by soluble molecules from pyroptotic cells (Fig. $6 \mathrm{c}, \mathrm{d}$ ), since there is no physical attachment between the pyroptotic $\mathrm{M} \phi$ and the normal $\mathrm{M} \phi$ in the co-culture system

In summary, this study demonstrates a novel mechanism by which NETs through the release of HMGB1 induce $\mathrm{M} \phi$ pyroptosis, which in turn plays an important role in directing the progress of inflammation following infection. These findings shed light on the proinflammatory role of NETs in augmenting inflammation in sepsis, and provide us with new information for generating therapeutic strategy against infectious diseases.

\section{Materials and methods \\ Animal strains}

All the mice used in the experiments were 8-10-weeksold and on a C57BL/6 background. C57BL/6 wild-type (WT) mice were purchased from the Jackson Laboratory (Bar Harbor, ME, USA). TLR4 knockout $\left(\mathrm{TLR}^{-1-}{ }^{-1}\right.$ mice, TLR9 knockout $\left(\right.$ TLR9 $^{-1-}$ ) mice, RAGE knockout $\left(\mathrm{RAGE}^{-1-}\right)$ mice, and HMGB1 knockout $\left(\mathrm{HMGB1}^{-1-}\right)$ 
mice were obtained from Dr. Timothy Billiar's laboratory at the University of Pittsburgh. All the animal experimental protocols were reviewed and approved by the Institutional Animal Care and Use Committees of University of Pittsburgh and VA Pittsburgh Healthcare System.

\section{Reagents}

Primary antibodies for cell staining and western blotting: ASC Ab (Santa Cruz Biotechnologies), rabbit polyclonal anti-mouse HMGB1 antibody (Abcam, Cambridge, MA, USA), rabbit polyclonal anti-mouse caspase-1 p10 (Santa Cruz Biotechnologies), and GAPDH (D16H11) XP Rabbit mAb (Cell Signaling Technology). Secondary antibodies including Alexa Fluor 488-conjugated antimouse IgG, Cy5-conjugated anti-mouse IgG, Alexa Fluor 488-conjugated anti-rabbit IgG, and Cy3-conjugated antirabbit IgG were provided by the Center for Biologic Imaging, University of Pittsburgh Medicine Center. In Situ Cell Death Detection Kit, TMR red (TUNEL) was purchased from Roche (Indianapolis, IN, USA). Annexin$\mathrm{V}$ detection kit was purchased from BD Biosciences. iScript $^{\text {tw }}$ Reverse Transcription Supermix and iTaq ${ }^{\text {Tx }}$ Universal SYBR ${ }^{\circledast}$ Green Supermix were purchased from BioRad. Phorbol 12-myristate 13-acetate(PMA) was from Sigma-Aldrich.

\section{Mouse model of CLP}

The mouse CLP model was carried out, as previously described $^{20}$. Mice were anesthetized with ketamine $(50 \mathrm{mg} / \mathrm{kg})$ and xylazine $(5 \mathrm{mg} / \mathrm{kg})$ via i.p. injection. After disinfection, a $1 \mathrm{~cm}$ midline laparotomy was made in the abdomen. The cecum was then exteriorized, and the distal end was ligated with a 6.0 silk suture and punctured once with a needle (21-gauge) to achieve a sublethal sepsis model. Mice were resuscitated with $(5 \mathrm{ml} / 100 \mathrm{~g})$ saline, and killed at different time points after surgery to retrieve the peritoneal lavage.

\section{BMDM isolation and culture}

The femurs and tibias were harvested from the WT or gene knockout mice, followed by the bone marrow being flushed with prechilled Dulbecco's modified Eagle's medium (DMEM).$^{34}$ Briefly, the cell pellets were collected by centrifugation at $4{ }^{\circ} \mathrm{C}$, and the erythrocytes were lysed with RBC lysis buffer (Thermo Fisher Scientific). The resultant cells were then washed two times with phosphate-buffered saline (PBS) and suspended in the cell culture medium (DMEM containing 10\% fetal bovine serum (FBS) complemented with $50 \mu \mathrm{g} / \mathrm{ml}$ penicillin/ streptomycin and $10 \mathrm{ng} / \mathrm{ml}$ recombinant macrophagecolony stimulating factor (Sigma-Aldrich, St. Louis, MO, USA)) at a concentration of $1 \times 10^{6}$ cells $/ \mathrm{ml}$ and seeded into 6-cm ultra-low attachment surface plates (Corning
Costar, Corning, NY, USA). The BMDM culture medium was changed on day 3 and day 5. BMDM were entirely differentiated and ready for use at day 7 .

\section{PMNs isolation and NETs induction}

PMNs were induced in the peritoneal cavity of the mice, as previously described. ${ }^{20}$ Briefly, mice were injected intraperitoneally with $1 \mathrm{ml} 9 \%$ casein solution twice overnight. The mice were killed $3 \mathrm{~h}$ after the second injection to harvest PLF. PLF was subsequently centrifuged, and the cell pellets were washed. The PMNs were isolated by discontinuous density gradient centrifugation with two commercially available solutions (Histopaque-1077 and Histopaque-1119) of differential density purchased from Sigma (St. Louis, MO), according to the manufacturer's instructions. PMNs $\left(10^{6}\right.$ cells $\left./ \mathrm{ml}\right)$ were incubated with $50 \mathrm{nM}$ PMA to induce NET formation.

\section{NET quantification assay}

The PMNs were cultured in 96-well plates at a concentration of $10^{6}$ cells $/ \mathrm{ml}$. At the indicated time points after treatment, $1 \mathrm{U} / \mathrm{ml}$ micrococcal nuclease (New England Biolabs, Ipswich, MA) was added. PMNs were incubated at $37^{\circ} \mathrm{C}$ for $15 \mathrm{~min}$ to allow the extruded DNA to detach from the cell debris. Cells were then centrifuged at $1800 \mathrm{~g}$ for $10 \mathrm{~min}$. Cell-impermeable DNA-binding dye SYTOX Green (Thermo Fisher Scientific, Waltham, MA) was added to the extracted supernatants and incubated in the dark for $15 \mathrm{~min}$. Extracellular DNA content is represented by the mean fluorescence intensity (MFI) detected with SpectraMax M2 (excitation wavelength $485 \mathrm{nM}$ and emission wavelength $530 \mathrm{nM}$ ).

\section{Western blot}

The supernatant of PLF or cell culture medium was concentrated 20 times by Ultracel- 3 membrane $3 \mathrm{kDa}$, and the BMDM lysates were separated by $8 \%$ and $15 \%$ sodium dodecyl sulfate polyacrylamide gel electrophoresis, and then transferred onto the PVDF membranes. After blocking for $1 \mathrm{~h}$ at room temperature with blocking buffer (LI-COR Biosciences, Lincoln, NE, USA), the blots were incubated with the primary antibody at $4{ }^{\circ} \mathrm{C}$ overnight, followed by incubation with appropriate secondary antibodies (LI-COR Biosciences) for $1 \mathrm{~h}$. Protein bands were detected using the Odyssey System from LI-COR Biosciences, and the intensity of each band was quantified using Image version $1.50 \mathrm{i}$. The intensity of the target protein band was normalized with a reference protein band and calculated for the fold changing.

\section{Detection of lysosome rupture and CatB activation \\ PM $\phi / B M D M\left(5 \times 10^{5}\right.$ cells $)$ cultured in $35 \mathrm{~mm}$ Petri dishes were stimulated with NETs $(200 \mu \mathrm{l})$ for $9 \mathrm{~h}$,}


followed by co-culturing with DQ Ovalbumin $(10 \mathrm{mg} / \mathrm{ml}$; Molecular Probes) or Magic Red CatB assay reagent $(10 \mathrm{mg} / \mathrm{ml}$; Immunochemistry Technologies, Bloomington, MN, USA) at $37^{\circ} \mathrm{C}$ for $1 \mathrm{~h}$ for the detection of lysosome rupture and $\mathrm{CatB}$ activation. After fixation with $4 \%$ paraformaldehyde, the cells were visualized by confocal microscopy. Cells were randomly selected for the measurement of intracellular fluorescence intensity by using Olympus FV10-ASW software (Olympus).

\section{Flow cytometry analysis of programmed cell death and pyroptosis}

Programmed cell death was analyzed by flow cytometry with apoptosis detection kit (BD Biosciences, Franklin Lakes, NJ). The peritoneal macrophages (PMs)/ BMDM were centrifuged, washed twice with prechilled PBS, and resuspended in the binding buffer. PMs/ BMDM were incubated with Annexin-V and 7-AAD for $15 \mathrm{~min}$ at room temperature in the dark, and then were analyzed by flow cytometry. The cells double-stained positive for Annexin-V and 7-AAD were considered to be programmed cell death. Cell pyroptosis was detected by two-color flow cytometry. PMs or BMDM were incubated with Alexa Fluor 488- labeled caspase-1 FLICA at $37^{\circ} \mathrm{C}$ for $1 \mathrm{~h}$. After being fixed with $4 \%$ paraformaldehyde, the PMs/BMDM were stained with TMR red-labeled In-Situ Cell Death Detection reagent (Roche Applied Science, Indianapolis, IN, USA), following the manufacturer's instructions. The cells were then analyzed by flow cytometry. The double-stained cells were identified as pyroptotic cells. Background and autofluorescence were determined by a control antibody with the same isotype staining. Acquisition was performed on 10,000 events using a FACScalibur cytometer (BD Biosciences, San Jose, CA, USA), BD LSR II (BD Biosciences) and FlowJo-V10 software (Tree Star, Ashland, OR, USA). The rate of cell death was calculated as (dead cells/total cells) $\times 100 \%$.

\section{$\mathbf{M} \phi-\mathbf{M} \phi$ co-incubation}

M $\phi-M \phi$ co-incubation was performed using Transwell plates (Corning Incorporated Life Sciences, Acton, MA, USA). BMDM $\left(5 \times 10^{5}\right.$ cells per well $)$ were plated and cultured in the top well of the Transwell, then treated with NETs for $18 \mathrm{~h}$ to induce pyroptosis, followed by transferring from the top well into a new Transwell where untreated BMDM were cultured in the bottom well. The co-cultures were then incubated for $6 \mathrm{~h}$ in DMEM containing $10 \%$ FBS.

\section{RNA extraction and quantitative real-time PCR}

The BMDM from the co-culture bottom wells were harvested, and total RNA was isolated by using TRIzol RNA Isolation Reagent (Thermo Fisher Scientific), following the manufacturer's instruction. Quantitative real-time PCR was done using iTaq Universal SYBR Green Supermix (1725121, Bio-Rad) in a Bio-Rad iQ5 real-time PCR machine (Bio-Rad). The specific primers for mouse TNF- $\alpha$ and IL-1 $\beta$ were also purchased from Bio-Rad: TNF- $\alpha$ forward, 5'-GACGTGGAACTGGCAGAAGAG-3' and reverse, $5^{\prime}$ TTGGTGGTTTGTGAGTGTGAG-3'; IL-1 $\beta$ forward, $5^{\prime}$ GAAATGCCACCTTTTGACATG-3' and reverse, 5' TGGATGCTCTCATCAGGACAG-3'. After the amplification protocol was over, PCR product was subjected to melt curve analysis using Bio-Rad iQ5 software. The fold change was calculated using the $\Delta \Delta C_{\mathrm{T}}$ threshold cycle method $^{35}$ and the value for the GAPDH gene, which was normalized to untreated groups.

\section{Data presentation and statistical analysis}

The data are presented as mean \pm S.E.M. of the indicated number of experiments. SPSS 19.0 was used for statistical analysis. Significances between groups were determined by using one-way ANOVA or two-tailed Student's $t$-test. $P<.05$ was considered as statistically significant.

\section{Acknowledgements \\ This work was supported by the National Institutes of Health Grant HL-079669 (to J.F.), National Institutes of Health Grant HL-123882 (to J.F.), National Institutes of Health Grant HL076179 (to J.F.), VA Merit Award 1I01BX002729 (to J.F.), National Natural Science Foundation of China 81470262 (to J.F.), National Natural Science Foundation of China 81400056 (to Y.Z.), Shanghai Municipal Commission of Health and Family Planning 201540161 (to Y.Z.), Shanghai Rising-Star Program 14QA1403200 (to P.Z.), and Shanghai Medical New Excellent Youth XYQ2013115 (to P.Z.).}

\section{Author details}

'Department of Thoracic Surgery, Shanghai Pulmonary Hospital, Tongji University School of Medicine, Shanghai 200433, China. ${ }^{2}$ Department of Surgery, University of Pittsburgh School of Medicine, Pittsburgh", PA 15213, USA. ${ }^{3}$ Department of Thoracic and Cardiovascular Surgery, The Children's Hospital of Zhejiang University School of Medicine, Hangzhou, Zhejiang China 310052. ${ }^{4}$ Research and Development, Veterans Affairs Pittsburgh Healthcare System, Pittsburgh, PA 15240, USA. ${ }^{5}$ McGowan Institute for Regenerative Medicine, University of Pittsburgh, Pittsburgh, PA 15219, USA

\section{Author contributions}

L.C, Y.Z., P.Z., and Y.L. planned and did experiments including cell isolation and treatment, confocal microscopy, western blotting, and flow cytometry; L.C., Y. Y., and D.L. did animal experiments; K.F., G.J., and J.F. planned the project and conceived the experiments; L.C., G.J., and J.F. conceived the data and wrote the manuscript.

\section{Conflict of interest}

The authors declare that they have no conflict of interest.

\section{Publisher's note}

Springer Nature remains neutral with regard to jurisdictional claims in published maps and institutional affiliations.

Received: 11 January 2018 Revised: 14 March 2018 Accepted: 27 March 2018

Published online: 22 May 2018 


\section{References}

1. Serafini, N. et al. The TRPM4 channel controls monocyte and macrophage, but not neutrophil, function for survival in sepsis. J. Immunol. 189, 3689-3699 (2012).

2. Serbina, N. V., Jia, T., Hohl, T. M. \& Pamer, E. G. Monocyte-mediated defense against microbial pathogens. Annu. Rev. Immunol. 26, 421-452 (2008).

3. Fan, J., Li, Y., Vodovotz, Y., Billiar, T. R. \& Wilson, M. A. Neutrophil NAD(P)H oxidase is required for hemorrhagic shock-enhanced TLR2 up-regulation in alveolar macrophages in response to LPS. Shock 28, 213-218 (2007).

4. Fan, J. \& Malik, A. B. Toll-like receptor-4 (TLR4) signaling augments chemokineinduced neutrophil migration by modulating cell surface expression of chemokine receptors. Nat. Med. 9, 315-321 (2003).

5. Jiao, Y. et al. Frontline science: macrophage-derived exosomes promote neutrophil necroptosis following hemorrhagic shock. J. Leukoc. Biol. 103 , 175-183 (2017)

6. Fan, J., Li, Y., Vodovotz, Y., Billiar, T. R. \& Wilson, M. A. Hemorrhagic shockactivated neutrophils augment TLR4 signaling-induced TLR2 upregulation in alveolar macrophages: role in hemorrhage-primed lung inflammation. Am. J. Physiol. Lung Cell Mol. Physiol. 290, L738-L746 (2006).

7. Warnatsch, A., loannou, M., Wang, Q. \& Papayannopoulos, V. Neutrophil extracellular traps license macrophages for cytokine production in atherosclerosis. Science 349, 316-320 (2015).

8. Brinkmann, V. et al. Neutrophil extracellular traps kill bacteria. Science $\mathbf{3 0 3}$, 1532-1535 (2004)

9. Kaplan, M. J. \& Radic, M. Neutrophil extracellular traps: double-edged swords of innate immunity. J. Immunol. 189, 2689-2695 (2012).

10. Papayannopoulos, V. \& Zychlinsky, A. NETs: a new strategy for using old weapons. Trends Immunol. 30, 513-521 (2009).

11. Sorensen, O. E. \& Borregaard, N. Neutrophil extracellular traps - the dark side of neutrophils. J. Clin. Invest. 126, 1612-1620 (2016).

12. Lotze, M. T. \& Tracey, K. J. High-mobility group box 1 protein (HMGB1): nuclear weapon in the immune arsenal. Nat. Rev. Immunol. 5, 331-342 (2005).

13. $\mathrm{Xu}$, J. et al. Macrophage endocytosis of high-mobility group box 1 triggers pyroptosis. Cell Death Differ. 21, 1229-1239 (2014).

14. Yang, J. et al. Hemorrhagic shock primes for lung vascular endothelial cell pyroptosis: role in pulmonary inflammation following LPS. Cell Death Dis. 7, e2363 (2016)

15. Wallach, D., Kang, T. B., Dillon, C. P. \& Green, D. R. Programmed necrosis in inflammation: toward identification of the effector molecules. Science $\mathbf{3 5 2}$ aaf2154 (2016).

16. Geng, Y. et al. Heatstroke induces liver injury via IL-1 beta and HMGB1-induced pyroptosis. J. Hepatol. 63, 622-633 (2015).

17. Chan, F. K., Luz, N. F. \& Moriwaki, K. Programmed necrosis in the cross talk of cell death and inflammation. Annu. Rev. Immunol. 33, 79-106 (2015).
18. Yost, C. C. et al. Neonatal NET-inhibitory factor and related peptides inhibit neutrophil extracellular trap formation. J. Clin. Invest. 126, 3783-3798 (2016).

19. Rohrbach, A. S., Slade, D. J., Thompson, P. R. \& Mowen, K. A. Activation of PAD4 in NET formation. Front. Immunol. 3, 360 (2012).

20. $\mathrm{Xu}, \mathrm{F}$. et al. Aging-related Atg5 defect impairs neutrophil extracellular traps formation. Immunology 151, 417-432 (2017).

21. Knight, J. S. et al. Peptidylarginine deiminase inhibition is immunomodulatory and vasculoprotective in murine lupus. J. Clin. Invest. 123, 2981-2993 (2013).

22. Stehlik, C. et al. Apoptosis-associated Speck-like protein containing a caspase recruitment domain is a regulator of procaspase-1 activation. J. Immunol. 171, 6154-6163 (2003).

23. Luo, L. et al. Proinflammatory role of neutrophil extracellular traps in abdominal sepsis. Am. J. Physiol. Lung Cell Mol. Physiol. 307, L586-596 (2014).

24. Soehnlein, O. \& Lindbom, L. Phagocyte partnership during the onset and resolution of inflammation. Nat. Rev. Immunol. 10, 427-439 (2010).

25. Saffarzadeh, M. et al. Neutrophil extracellular traps directly induce epithelial and endothelial cell death: a predominant role of histones. PloS ONE 7, e32366 (2012).

26. Nakazawa, D. et al. Histones and neutrophil extracellular traps enhance tubular necrosis and remote organ injury in ischemic AKI. J. Am. Soc. Nephrol. 28 1753-1768 (2017).

27. $\mathrm{Hu}, \mathrm{Z}$. et al. Neutrophil extracellular traps induce $\mathrm{IL}-1 \beta$ production by macrophages in combination with lipopolysaccharide. Int. J. Mol. Med. 39, 549-558 (2017).

28. Kumar, S. V. et al. Neutrophil extracellular trap-related extracellular histones cause vascular necrosis in severe GN. J. Am. Soc. Nephrol. 26 2399-2413 (2015)

29. Park, J. S. et al. Involvement of toll-like receptors 2 and 4 in cellular activation by high mobility group box 1 protein. J. Biol. Chem. 279, 7370-7377 (2004).

30. Ivanov, S. et al. A novel role for HMGB1 in TLR9-mediated inflammatory responses to CpG-DNA. Blood 110, 1970-1981 (2007).

31. Huebener, P. et al. The HMGB1/RAGE axis triggers neutrophil-mediated injun amplification following necrosis. J. Clin. Invest. 125, 539-550 (2015).

32. Bergsbaken, T., Fink, S. L. \& Cookson, B. T. Pyroptosis: host cell death and inflammation. Nat. Rev. Microbiol. 7, 99-109 (2009).

33. Shi, J. et al. Cleavage of GSDMD by inflammatory caspases determines pyroptotic cell death. Nature 526, 660-665 (2015).

34. Weischenfeldt, J., Porse, B. Bone marrow-derived macrophages (BMM): isolation and applications. CSH Protocols 2008, 1-6 (2008).

35. Livak, K. J. \& Schmittgen, T. D. Analysis of relative gene expression data using real-time quantitative PCR and the 2(-Delta Delta C(T)) Method. Methods 25, 402-408 (2001) 